\title{
TAREAS DE ORIENTACIÓN Y APRENDIZAJES TRANSVERSALES
}

\author{
ORIENTATION TASKS AND TRANSVERSAL LEARNING
}

https://doi.org/10.15304/ie.30.7062

\author{
José María Fernández Batanero \\ Universidad de Sevilla \\ batanero@us.es
}

\section{RESUMEN}

Los aprendizajes transversales contribuyen a dar significatividad y funcionalidad desde la conexión de los conocimientos disciplinares con los temas y contextos sociales, culturales y éticos presentes en el entorno inmediato del alumnado. Por lo tanto, el saber, el hacer y el ser en torno al medio ambiente, la salud, la convivencia democrática, la afectividad y sexualidad, entre otros, son aprendizajes integrales que permiten el pleno desarrollo como personas individuales y sociales. Su puesta en práctica va a implicar, entre otros aspectos, la presencia de la orientación y la acción tutorial como un factor de calidad y como parte fundamental de la función docente. En el presente trabajo recogemos, por un lado, los principios básicos y objetivos de la orientación y la acción tutorial con respecto a los aprendizajes transversales, así como las competencias genéricas y específicas para orientadores y tutores. Por otro, se establecen pautas sobre el papel de la orientación en la elaboración de proyectos educativos transversales, terminando con algunas consideraciones a cerca de la Educación para la Salud, como contenido transversal actual y global. Palabras clave: aprendizaje transversal, orientación educativa, tutoría, competencias docentes.

\begin{abstract}
Transversal learning contributes to give significance and functionality from the connection of disciplinary knowledge with the social, cultural and ethical issues and contexts present in the immediate environment of the students. Therefore, knowing, doing and being around the environment, health, democratic coexistence, affectivity and sexuality, among others, are integral learning that allows full development as individual and social persons. Its implementation will imply, among other aspects, the presence of guidance and tutorial action as a quality factor and as a fundamental part of the teaching function. In this work we collect, on the one hand, the basic principles and objectives of guidance and tutorial action with respect to transversal learning, as well as generic and specific competences for counselors and tutors. On the other, guidelines are established on the role of guidance in the development of transversal educational projects, ending with some considerations about education for health, as current and global transversal content. Keywords: Cross-learning, educational guidance, tutoring, teaching skills.
\end{abstract}




\section{INTRODUCCIÓN}

A lo largo de los últimos años hemos venido asistiendo a una serie de cambios que de manera progresiva y a todos los niveles (económico, político, social, educativo, etc.), se están produciendo en la sociedad. Transformaciones que son consecuencia de los progresos alcanzados en los diferentes campos científicos y que han dado lugar a la llamada "sociedad del conocimiento y la información" en la que nos encontramos. La educación, no es ajena a estos avances, progresos y transformaciones, y debe responder de forma eficaz a las demandas actuales que le exige la sociedad.

Si echamos una mirada hacia atrás, vemos como desde 1990, con la entrada en vigor de la Ley Orgánica de Ordenación General del Sistema Educativo (LOGSE) se ha intentado en mayor o menor medida dar respuesta desde la escuela a los problemas de la sociedad. Para ello, aparecen los llamados temas transversales del currículum (Educación para la paz, Educación para la igualdad de oportunidades, Educación para la Salud, Coeducación, Educación Ambiental, Educación para el Consumidor, Educación Vial, Educación Moral y Cívica...). Ello constituyó el mayor símbolo de innovación y de apertura de la escuela a la sociedad en aquel momento, utilizándose incluso como el paradigma de la Reforma que se quería emprender.

En la exposición de motivos de la citada Ley Orgánica, así como en sus artículos primero y segundo, se hablaba de:

Sentido personal y moral; generar actitudes y hábitos individuales y colectivos; valores con los que nos identificamos individual y colectivamente; respeto de los derechos y libertades; ejercicio de la tolerancia; formación para la paz y la solidaridad; formación en la igualdad entre los sexos y rechazo a todo tipo de discriminación.

Si bien, la designación temas transversales está en desuso y se ha ido sustituyendo por "Tratamiento transversal de la educación en valores" (en el art. 121 de la LOE-LONCE) o "elementos transversales" (art. 10 del Real Decreto 126/2014).

Estos ámbitos pretenden conseguir la formación integral del alumnado, de transmitir la cultura y contribuir al desarrollo de la ciencia y la investigación. Así mismo, en la actualidad deben contribuir a la formación de personas que sean capaces de convivir en la llamada sociedad de la información y comunicación, donde la globalización, la accesibilidad a la información, la internacionalización de la economía, la inmigración, la pobreza, la contaminación, etc., suponen retos educativos.

Entren las características principales de estos "temas transversales" vs "elementos transversales" se encuentran las siguientes: recogen problemas de relevancia social; se basan en las demandas que la sociedad hace a la escuela; alto poder de renovación de los contenidos; componente funcional y actitudinal; favorecen la motivación y aprendizaje, por conectar con los intereses del alumno; reciben un tratamiento multidisciplinar o interdisciplinar; responden a principios didácticos que dan carácter y orientan el currículo; ofrecen grandes posibilidades educativas por el interés que suscitan entre el alumnado; etc. 
Todos estas características y factores que definen la nueva realidad de la educación suponen: una docencia personalizada, es decir, centrada en el estudiante y adaptadas a sus intereses; una formación a lo largo de la vida, orientada a la consecución de competencias claves para desenvolverse en una sociedad democrática, resaltando el papel que van a tener las Tecnologías de la Información y la Comunicación (TIC) en los procesos de aprendizaje del alumnado. Todo ello va a implicar, entre otros aspectos, la presencia de la orientación y la acción tutorial como un factor de calidad y como parte fundamental de la función docente. En este sentido, la orientación y la acción tutorial se convierten en una herramienta imprescindible, al ser entendida como un proceso de ayuda para la formación del estudiante en todas sus dimensiones: personal, académica y profesional (Amor, 2016).

En definitiva, una docencia de calidad implica necesariamente una redefinición del trabajo del profesorado, donde su nuevo rol sea de creador y organizador de ambientes de aprendizaje, implicando al alumnado en su propio proceso de aprendizaje a través de las estrategias y actividades adecuadas (Herrera et al., 2006). Una educación de calidad implica también la introducción de valores que nos permitan reflexionar sobre la dimensión más personal de la educación.

\section{ORIENTACIÓN EDUCATIVA, APOYO A LA FUNCIÓN TUTORIAL Y APRENDIZAJES TRANSVERSALES}

Como hemos comentado anteriormente los aprendizajes transversales contribuyen a dar significatividad y funcionalidad desde la conexión de los conocimientos disciplinares con los temas y contextos sociales, culturales y éticos presentes en su entorno. Por lo tanto, el saber, el hacer y el ser en torno al medio ambiente, el autocuidado y la prevención, la convivencia democrática, la afectividad y sexualidad, entre otros, son aprendizajes integrales que permiten el pleno desarrollo como personas individuales y sociales.

Es en este contexto donde el docente se convierte en un mediador de estos aprendizajes y los estudiantes. Diagnosticando sus necesidades, preparando y organizando las clases mediante la realización de actividades (individuales y cooperativas) de gran potencial didáctico y que consideren las características de los estudiantes. Buscando y preparando materiales y recursos, despertando el interés de estos (el deseo de aprender) hacia los objetivos y contenidos didácticos (establecer relaciones con sus experiencias vitales, con la utilidad que obtendrán...). Cultivando y potenciando la diversidad, investigando en el aula, colaborando en la gestión del centro educativo, ofreciendo tutoría, etc.

En el desarrollo de todas las funciones demandadas al profesor la orientación y la acción tutorial se convierten en una herramienta imprescindible para alcanzar los objetivos de la educación. Desde esta óptica la orientación y la acción tutorial se entienden como un proceso de ayuda para la educación del estudiante en todas sus dimensiones: personal, académica y profesional. Se concibe como una actividad académica vinculada institucionalmente e integrada en la práctica docente del profesorado, desde lo más próximo al alumnado y con una perspectiva multidimensional de su formación. 
En esta línea, la tutoría y la orientación deberán:

- Establecer diferentes formas de seguimiento de los aprendizajes de los estudiantes individualmente, proporcionando feed-back.

- Ayudar al alumnado en la selección de actividades de formación adecuadas a sus circunstancias.

- Favorecer el uso de las Tecnologías de la Información y Comunicación (TIC) con el objetivo de mejorar la acción tutorial: bases de datos para el seguimiento de los estudiantes, tutorías telemáticas....

- Dar ejemplo en la selección y buen uso de los recursos tecnológicos utilizándolos (como instrumento didáctico y como recurso de trabajo en general) solamente cuando (y de la manera que) aporten ventajas sobre el empleo de otros materiales más asequibles.

- Dar ejemplo en la organización de los recursos tecnológicos (a nivel personal, clase, centro...)

- Ser el responsable de iniciar y mantener los contactos con las familias del alumnado.

- Ser un ejemplo para los estudiantes.

\subsection{Principios básicos y objetivos para la acción del tutor en su desempeño ante aprendizajes transversales}

Los principios, que se detallan a continuación, representan la aplicación de las experiencias previas y de otros modelos educativos analizados a las condiciones específicas de la institución:

\section{Potenciar la autonomía del alumno}

El alumno es autónomo y la principal tarea de la acción orientadora es contribuir a ello, mediante la estimulación del sentido de la responsabilidad, implementando una constante tutorización para que los estudiantes sean capaces de asimilar los errores, superándolos de forma positiva y afianzando su autoestima.

\section{Provocar el aprendizaje mediante el fomento de la curiosidad}

El tutor tiene como objetivo provocar que el alumnado alcance lo mejor de sí mismos. Por ello tienen la función de brindar a los estudiantes todas las herramientas posibles para alcanzar sus objetivos, favoreciendo la curiosidad. Cuando los alumnos sienten curiosidad es más fácil que se interesen en un tema y aprendan. La química de nuestro cerebro se modifica cuando estamos en un estado de curiosidad. La curiosidad nos lleva a cuestionar las cosas, a buscar las preguntas adecuadas para descubrir las mejores respuestas. Crear un ambiente que abra las mentes para preguntarse los porqués.

La curiosidad es la llave que abre las ventanas de la atención, y con ella el aprendizaje y la memoria. 


\section{Fomentar la formación}

Es desde lo académico que buscamos promover la formación integral de nuestros estudiantes y desarrollar articuladamente las competencias específicas y transversales que permitirán a nuestros estudiantes a adquirir los saberes que se recogen en el Informe de la Unesco, $L a$ educación encierra un tesoro (Delors, 1996), en el que se ponían encima de la mesa las principales y más urgentes cuestiones sobre la educación para el siglo XXI, y el papel que la misma iba a jugar para las generaciones futuras. Es decir, aprender a conocer el mundo, a hacer, a vivir juntos y a ser.

\section{Avivar la cooperación}

Se trata de una responsabilidad compartida en la que se compromete todo el equipo docente que trabaja con un grupo de alumnos, coordinada por el Tutor y apoyada por el Departamento de Orientación del centro y/o los Servicios de Orientación y Apoyo del sector.

\section{Integrada en la función docente}

Se trata de potenciar la integración de la acción tutorial en el currículo, y que, por lo tanto, esté contemplada en la selección y secuenciación de los contenidos y en el diseño de las actividades de enseñanza-aprendizaje y de la evaluación.

\section{Potenciar la intervención social}

Todas las personas implicadas en las labores tutoriales (incluido el alumno), deben de intervenir en el contexto educativo y social para que también en éste se den cambios que favorezcan las mayores y mejores cotas de realización personal y grupal (Olmedo y Pegalajar, 2014).

La intervención social exige compromiso social, y éste surge como el pilar en el que se sustenta el aprendizaje, ya que constituye la base de las políticas educativas, para contribuir a la mejora y al progreso social, sin olvidarse de aunar la equidad y la calidad. Este compromiso social conlleva establecer fuertes mecanismos de participación y colaboración entre las instituciones educativas y los contextos de pertenencia; por lo que se crean así auténticas comunidades de aprendizaje (Domene, Martín Gutiérrez y Morales Lozano, 2019).

Los principios citados orientan el objetivo principal de la orientación académica en el área transversal que se centra en desarrollar en los estudiantes las competencias transversales necesarias para una formación integral. Como medio para alcanzar este objetivo se emplea acompañamiento socio-afectivo y académico a los estudiantes contribuyendo a su formación integral, orientando su proceso de desarrollo autónomo en una dirección beneficiosa para ellos, teniendo en cuenta al alumno como ser con derecho a la búsqueda del mejor camino para su desarrollo personal (Pámies y Galindo, 2015). 
Son aprendizajes que tienen un carácter comprensivo y general, y apuntan al desarrollo personal, ético, social e intelectual de los estudiantes.

No se consiguen por medio de un sector de aprendizaje en particular, sino que dependen del conjunto del currículum y de las distintas experiencias escolares.

Deben ser promovidos a través de las diversas disciplinas y en las distintas dimensiones del quehacer educativo.

Tabla 1. Características de los objetivos de aprendizajes transversales

\subsection{Competencias genéricas y específicas para orientadores y tutores}

Es necesario señalar, la existencia de diversas taxonomías sobre las competencias (Bisquerra y Pérez Escoda, 2007), entre otras, se pueden señalar las competencias técnicas, competencias profesionales, competencias participativas, competencias personales, competencias básicas, competencias clave, competencias transversales, competencias genéricas, competencias emocionales, competencias socioemocionales, etc. Según Bisquerra y Pérez Escoda (2007) la mayoría de estas clasificaciones incluyen diferentes clases de competencias y destacan fundamentalmente dos tipos de dimensiones: competencias de carácter técnico-profesional y competencias de carácter sociopersonal. Por un lado, las competencias de carácter técnico-profesional versan sobre conocimientos y procedimientos en relación con un determinado ámbito profesional o de especialización e incluyen el saber y el saber hacer indispensables para el desempeño experto de una actividad laboral. Por otro, las competencias socio-personales (competencias emocionales) están relacionados con conocimientos y procedimientos intrapersonales (automotivación) e interpersonales (resolución de conflictos).

Por otro lado, ya hace casi tres décadas que la Asociación Internacional para la Orientación Educativa y Profesional (AIOEP) se interesó por el desarrollo de una serie de competencias internacionales que los profesionales necesitan para proporcionar servicios de orientación educativa y profesional, con garantía de un servicio profesional de calidad. La serie de competencias fue validada por una bolsa de 700 profesionales que trabajan en diferentes empleos, de 38 países. Dichas competencias fueron las siguientes (Gamboa, Ortega, Cerdas, Fallas, y Vargas, 2010):

\section{Competencias centrales (genéricas)}

- Demostrar profesionalidad y comportamiento ético en el desempeño de sus tareas.

- Promover en los clientes el aprendizaje y el desarrollo personal y de la carrera.

Apreciar y atender las diferencias culturales de los clientes, posibilitando la interacción efectiva con poblaciones diversas.

— Integrar la teoría y la investigación en la práctica de la orientación. 
- Poseer habilidad para diseñar, implementar y evaluar intervenciones y programas de orientación.

- Ser consciente de las propias capacidades y limitaciones.

- Mostrar habilidad para usar el nivel apropiado de lenguaje para comunicarse con colegas o clientes, logrando así una comunicación eficaz.

- Tener conocimiento de información actualizada sobre educación, formación, tendencias de empleo, mercado de trabajo y asuntos sociales.

- Demostrar sensibilidad social e inter-cultural.

- Mostrar habilidad para cooperar de manera eficaz con un grupo de profesionales.

- Demostrar conocimiento sobre el desarrollo evolutivo de la persona.

Más recientemente, en un estudio realizado por Cejudo (2017), en relación con la importancia de las competencias profesionales a las que los orientadores conceden mayor importancia en orden a formar un orientador altamente competente, se resaltaban las siguientes: las competencias de gestión de la convivencia, las competencias de trabajo en equipo, las competencias socio-relacionales, las competencias de fomento de la innovación educativa, las competencias emocionales, las competencias comunicativas, las competencias metodológicas, las competencias científicas, las competencias organizativas y, por último, las competencias digitales. Cabe destacar que todas las competencias profesionales recogidas en la EICPO se consideran con una importancia entre alta y muy alta. Llama la atención la ausencia de competencias transversales, pero ello puede ser debido a la no inclusión de estas competencias en el cuestionario original.

Las competencias del tutor deben de enfocarse en tres direcciones:

- Nuevas habilidades. Manejo del discurso electrónico, planificación de actividades individuales y trabajo en grupo, adecuándose a la diversidad del alumnado; generar propuestas que favorecen el conocimiento mutuo y la información de una comunidad de aprendizaje; seguimiento, supervisión y evaluación del aprendizaje en línea.

- Nuevos conocimientos: Nuevos componentes que generen propuestas que favorezcan el conocimiento mutuo y la conformación de una comunidad de aprendizaje; dominios tecnológicos de e-learning; teorías del aprendizaje; conocimiento pedagógico, tecnológico y disciplinar (TPACK).

- Actitudes: cordialidad, accesibilidad, capacidad de aliento; actitud de interés hacia lo que dice y escribe el alumno; empatía; capacidad de motivación; compromiso y dedicación a la tarea, capacidad para anticipar conflictos y resolverlos con inteligencia, capacidad para liderar de forma democrática. 


\section{EL PAPEL DE LA ORIENTACIÓN EN LA ELABORACIÓN DE PROYECTOS EDUCATIVOS TRANSVERSALES}

El Proyecto Educativo aparece y se entiende como uno de los elementos esenciales en el que se manifiesta el ejercicio de la autonomía pedagógica de los centros docentes, constituyendo el instrumento que hace explícito el estilo educativo de un centro docente en una realidad concreta, dándole coherencia y continuidad. El Proyecto Educativo de los centros docentes es uno de los elementos que integran el Plan de Centro. En este documento cada centro educativo expresa y concreta el tipo y finalidad de la educación que desea llevar a cabo y que va a desarrollar dentro, y en función, de las condiciones concretas y peculiares de su contexto. En este sentido, deberá contemplar los valores, los objetivos y las prioridades de actuación del centro en su conjunto, no limitándose sólo a los aspectos puramente curriculares, sino también a aquellos otros que, desde un punto de vista cultural y social, hacen de cada centro docente un elemento dinamizador de la zona donde está ubicado. La transversalidad introduce la educación en valores imprescindibles para producir comportamientos democráticos y pluralistas.

Son muchos los aspectos del Proyecto Educativo en los que el papel de la orientación educativa puede realizar importantes aportaciones y sugerencias cumpliendo con su función asesora. En primer lugar, hay que orientar el Proyecto Educativo hacia la definición de unas líneas generales de actuación pedagógica que permitan al centro docente educar desde la diversidad y para la igualdad, en un adecuado clima de convivencia y respeto.

Los responsables de la orientación educativa cuentan con grandes habilidades y recursos para conseguir que este documento tenga una utilidad vital y real para el trato de la convivencia en los centros docentes. La programación curricular de contenidos transversales ofrece una oportunidad extraordinaria para el desarrollo de aprendizajes significativos. El papel de la orientación es fundamental a la hora de asesorar en la elaboración de propuestas transversales acordes con los problemas del entorno inmediato del alumnado. Para ello es necesario que los orientadores partan de estas premisas (Domingo, Fernández Gálvez y Barrero, 2016).

\section{a) Poner en valor la acción sobre el currículum}

Es necesario trabajar sobre el currículum, las decisiones curriculares y las dimensiones colaterales que puedan influir en él. Si se lograra dar un giro en la priorización de ámbitos hacia el currículum podrían ser más efectivos e incidir en aspectos esenciales y preventivos con el mismo esfuerzo. La orientación es un factor de calidad para mejorar los resultados de los estudiantes, tanto en los niveles académicos como en la educación en valores, propios de la educación integral.

\section{b) Asumir responsabilidad en el currículum con visión sistémica}

Hay que ver más allá de lo que programamos desde las diferentes áreas y niveles, tener visión global. Los aprendizajes transversales exigen una visión sistémica, donde la globalización y la interdisciplinariedad juegan un importante papel. 


\section{c) Lineas de coherencia}

Desde los contenidos a metodología y de ahí a evaluación y a resultados en contenidos, en aprendizajes... Esta es la verdadera innovación y que tenga todo sentido para el alumnado y profesorado.

\section{d) Con planteamientos curriculares transversales}

Muchos profesores solo ven el currículum de su materia y desde su materia. Les cuesta mucho hacer inferencias y relaciones con otras materias y no tiene una mirada sobre el currículum integral, los elementos curriculares que hacen que sea posible atender a la persona en su conjunto o que se operen procesos capaces de alcanzar competencias.

\section{e) Partiendo de un paradigma emergente: la educación global}

Como ha señalado Cloud (2014), el currículo actual está desconectado de la realidad, es decir, de las grandes problemáticas sociales y ambientales que hoy en día zarandean nuestro interconectado y desequilibrado mundo. La educación global trata de dotar de forma integrada al alumnado con las competencias claves para desenvolverse con acierto y eficacia en un mundo "plano", dinámico e interdependiente (Darling-Hammond, 2010). Este emergente enfoque pedagógico pretende dotar a los ciudadanos del siglo XXI no sólo para que comprendan en profundidad su intrincada y mutante realidad, sino además para que se impliquen en su mejora en la medida de lo posible. En este emergente paradigma se intenta preparar a los estudiantes para afrontar los desafíos de la sociedad del conocimiento equipándolos con las necesarias e imprescindibles herramientas para ello (López Ruiz, 2019).

De este modo destaca la UNESCO (2016: 12) este enfoque más integral de concebir la educación global en un documento más reciente:

Se ha producido un cambio en el discurso y en la práctica de la educación. Este cambio reconoce la importancia de la educación y del aprendizaje para comprender y solucionar los problemas globales en los ámbitos social, político, cultural, económico y ambiental. El papel de la educación está llegando más allá del desarrollo de los conocimientos y las competencias cognoscitivas para la construcción de valores, competencias y actitudes entre los alumnos. Se espera que la educación facilite la cooperación internacional y promueva la transformación social en una forma innovadora para lograr un mundo más justo y pacífico, tolerante, inclusivo, seguro y sostenible.

\section{f) Involucrar a las familias}

Si queremos establecer un modelo educativo integral en las escuelas y potenciar en nuestros alumnos no solo su desarrollo cognitivo, también el afectivo y social, es necesario que las familias se involucren en el proceso de aprendizaje de estos. 


\section{LA EDUCACIÓN PARA LA SALUD. CONTENIDOTRANSVERSAL ACTUALY GLOBAL}

La Educación para la Salud está teniendo una gran importancia actualmente con la llegada del virus conocido como SARS-CoV-2, que produce la enfermedad llamada del Covid-19, declarada como pandemia por la Organización Mundial de la Salud (OMS) el 30 de enero 2020.

Fue uno de los grandes temas transversales con la LOGSE y en leyes posteriores. En el actual diseño curricular figura en el objetivo $\mathrm{k}$ de educación primaria y de manera implícita en las competencias clave. En el ámbito de los contenidos se la contempla en CC. Naturales y Educación Física, pero limitándolo a cuestiones de ejercicio y alimentación.

A lo largo de los últimos años, las administraciones educativas en nuestro país han abierto un amplio cauce de actuación para el desarrollo de la Promoción y EpS con las reformas llevadas a cabo, primero con la Ley Orgánica 1/1990 de Ordenación General del Sistema Educativo (LOGSE), y posteriormente con el desarrollo de la Ley Orgánica 2/2006 de Educación (LOE) y la Ley Orgánica 8/2013 para la Mejora de la Calidad Educativa (LOMCE). La necesidad de estas actuaciones ha sido recomendada desde diversas entidades nacionales e internacionales (OMS, UNESCO, Consejo de Europa, Comisión Europea, Ministerio de Sanidad y Consumo, Ministerio de Educación, etc.). Pero además su importancia en la escuela se ha puesto de manifiesto recientemente con la pandemia del COVID-19. La incorporación de las competencias básicas al currículo es una primera apuesta por acercar el sistema educativo español a las exigencias internacionales. En este sentido, será esencial la colaboración entre el personal sanitario y docente (Talavera y Gavidia, 2013) y que el profesorado cuente con conocimientos sobre los temas básicos de salud, competencias para desarrollar estos conocimientos y habilidades que puedan ser aprendidas (Gavidia, 1993; Oliva, 2011).

Sin embargo, y a pesar de los esfuerzos llevados a cabo por la Administración para desarrollar la transversalidad, incluida la Competencia en Educación para la Salud, que deben ser incorporadas en los proyectos curriculares, existe una duda razonable de su plena integración en la escuela, debido a las dificultades que ello supone (falta de motivación del profesorado, la ausencia de directrices metodológicas comunes para su incorporación en el currículo y la necesidad de concretar los contenidos en Promoción y EpS) (Talavera y Gavidia, 2007). Así, el currículo oficial de la Enseñanza Secundaria Obligatoria en el desarrollo de los Contenidos Mínimos por el Ministerio de Educación, aunque recoge parcialmente las recomendaciones en Educación para la Salud de los organismos expertos, no atiende de manera adecuada a las Competencias en Promoción de la Salud que deben adquirir los jóvenes españoles (Giménez, Fernández, Mayoral y Gavidia, 2016).

Ya en la conferencia Europea de Educación para la Salud de Dublín de 1990 se instaba a los sistemas educativos a que incluyeran en sus respectivos currículos escolares contenidos de Promoción y Educación para la Salud. Han pasado 30 años y dichos contenidos parecen que han ido perdiendo fuerza. De ahí la importancia de retomarlos y replantearlos de nuevo en esta sociedad que se ve abocada a amenazas continuas en el ámbito de la salud. 
Gavidia (2016) manifiesta que entre las primeras decisiones acerca de los contenidos formativos que deberían aprender los estudiantes para tener comportamientos saludables se encuentra el llevado a cabo por la Generalitat de Catalunya y que posteriormente fue tomado por el Gobierno Vasco como un modelo de enseñanza-aprendizaje de la Educación para la Salud en la Escuela. El modelo se basaba en un currículum establecido por una "Comisión de Expertos", que fijó unos objetivos y contenidos determinados para su trabajo en el aula. Por su parte, el gobierno de Aragón trabajo con un modelo de proyectos, donde la selección de objetivos y contenidos a trabajar, eran seleccionados mediante proyectos determinados a iniciativa de los grupos de trabajo interesados en una temática concreta en función de las necesidades educativas sentidas en su comunidad y en su entorno ambiental próximo.

Con posterioridad han sido muchas las actuaciones desarrolladas por las diferentes Administraciones Autónomas para desarrollar los diferentes conocimientos de la Educación para la Salud (Valderrama, Gavidia et al., 1997, Pérez de Eulate y Ramos, 2005, Gavidia, 2016).

Con la llegada del Espacio Europeo de Educación Superior (EEES) hace que el foco de atención se centre en las competencias a desarrollar y se dejen de un lado los contenidos de la materia. Estas competencias en Salud que tienen que adquirir el alumnado en la educación obligatoria estaban todavía por ser seleccionadas en 2015. Autores como Gavidia (2016) a través de un trabajo realizado en el proyecto subvencionado por el Ministerio de Economía y Competitividad español (MINECO) denominado "Competencias a adquirir por los jóvenes y el profesorado en Educación para la Salud durante la escolarización obligatoria", cuyo objetivo principal pretendía concretar las competencias en Salud que deben adquirir los ciudadanos españoles en su paso obligatorio por la Escuela y comprobar hasta qué punto las propuestas curriculares que realiza la administración las tienen en cuenta.

Para ello, se planteaban los siguientes interrogantes:

¿Se desarrollan las competencias en salud necesarias para construir un entorno más humano y saludable? ¿cuáles son esas competencias necesarias para un desarrollo saludable personal y social? Y... ¿Qué contenidos son precisos tratar en el aula para desarrollar esas competencias en salud? ¿tiene en cuenta el currículo escolar esta esfera de desarrollo personal y comunitario?

De forma general, el estudio establece que los problemas o situaciones de salud se pueden agrupar en los " 8 ámbitos de salud" siguientes: alimentación y Actividad física, Adicciones, Salud Mental y Emocional, Sexualidad, Accidentes, Higiene, Medio Ambiente y promoción de la Salud. Este último ámbito es transversal al resto y contiene situaciones relacionadas con los otros ámbitos.

Solo dando respuestas a estas preguntas la Educación para la Salud se convierte en un precioso y necesario instrumento de Promoción de la Salud y una herramienta clave de intervención de salud en estas edades. La educación es tarea de todos, por ello los centros docentes, junto con el hogar, son los dos lugares clave donde tiene lugar el desarrollo individual y social de las personas. Los centros educativos tienen la necesidad de incorporar los temas 
relacionados con la Salud como una de las bases que les permitirán alcanzar los objetivos educativos previstos y dar respuesta a uno de los grandes problemas de la sociedad.

Para ello, el Plan estratégico de salud escolar y estilos de vida saludable gira en torno a tres ámbitos: los aspectos psicológicos, los sociales y los educativos. Salud emocional, alimentación saludable y equilibrio con el entorno serán los tres aspectos que guían el Plan de salud escolar cuyas actuaciones sustentan los principios de sostenibilidad que promueve la Agenda 2030 europea. En esta línea, el Plan propone 3 objetivos fundamentales:

Fomentar y apoyar un modelo educativo dirigido al desarrollo de la salud integral de toda la comunidad educativa.

Proporcionar una formación adecuada a todos los miembros de la comunidad educativa.

Proporcionar un desarrollo vital saludable desde la perspectiva física, psicológica, social y medioambiental.

La consecución de estos objetivos se determina en cinco líneas de actuación:

Línea 1: establecer y reforzar marcos de colaboración institucional entrela Administración central y autonómica, y los agentes implicados para la promoción de estilos de vida saludable en el ámbito educativo.

Línea 2: diseñar estrategias de promoción de la salud en el ámbito educativo con la implicación de la comunidad educativa en el fomento y desarrollo de estilos de vida saludable.

Línea 3: crear y desarrollar la Red Estatal de Escuelas Promotoras de la Salud y favorecer la creación y mantenimiento de Escuelas y Redes de Escuelas Promotoras de Salud.

Línea 4: asegurar la formación y capacitación del profesorado para la educación y promoción de la salud.

Línea 5: investigar y evaluar los logros relacionados con la adquisición de estilos de vida saludable.

En lo que a nosotros nos atañe en el marco de la orientación y acción tutorial debemos destacar la línea 4, referida a la formación del profesorado. Para poder llevar a cabo una educación de calidad, el profesorado deberá desarrollar una serie de competencias docentes entendiendo estas como el conjunto de conocimientos, capacidades, actitudes, valores y creencias que son necesarias movilizar para la consecución de las tareas educativas.

El profesorado con formación y competencias necesarias para el desarrollo de la Educación para la Salud, está en condiciones de tener un mayor éxito. Autores como Riquelme (2006) consideran que alguno de los factores que no contribuyen al aprendizaje de dicho eje transversal han sido:

Programas que se desarrollan en respuesta a una crisis percibida puntual (especialmente si vienen acompañadas de tácticas y discursos alarmistas). 
Una amplia participación del centro de enseñanza, espasmódica y carente de coordinación.

Programas basados en gran medida en portavoces y recursos externos con poca implicación de la plantilla del centro de enseñanza.

Poca o ninguna inversión en la formación de los profesores y en la entrega de recursos de apoyo.

\section{REFERENCIAS BIBLIOGRÁFICAS}

Amor, M. I. (2016). Evaluación de la orientación y la Tutoría en la Facultad de Ciencias de la Educación de la Universidad de Córdoba. Educatio Siglo XXI, 34(1), 93-112. DOI: https://doi.org/10.6018/j/253231

Bisquerra, R. y Pérez Escoda, N. (2007). Las competencias emocionales. Educación XXI, 10(1), 61-82. DOI: https://doi.org/10.5944/educxx1.1.10.297

Cejudo, J. (2017). Competencias profesionales y competencias emocionales en orientadores escolares. Profesorado. Revista de Currículum y Formación del Profesorado, 21(3), 349-370. Disponible en https://recyt.fecyt.es/index.php/profesorado/article/view/59802

Cloud, J. P. (2014). Educar para un futuro sostenible. En H. H. Jacobs (Ed.), Currículum XXI. Lo esencial de la educación para un mundo en cambio. Madrid: Narcea.

Darling-Hammond, L. (2010). The flat world and education. How America's commitment to equity will determine our future. New York: Teachers College Press.

Delors, J., et. al. (1996): La Educación encierra un tesoro. Informe a la UNESCO de la Comisión Internacional sobre la educación para el Siglo XXI. Madrid: Santillana-UNESCO.

Domene, S., Martín Gutiérrez, A. y Morales Lozano, J. A. (2019). Colaborar y emprender. En J. M. Fernández Batanero y A. López Martínez (Coords.). Nuevos horizontes en educación. Innovaciones y experiencias (pp. 67-86). Barcelona: Octaedro.

Domingo, J., Fernández Gálvez, J. y Barrero, B. (2016). El orientador ante el reto de la mejora curricular. Un estudio de caso. Revista Electrónica de Investigación Educativa, 18(2), $27-$ 39. Disponible en http://redie.uabc.mx/redie/article/view/966

Gamboa, A., Ortega, J., Cerdas, V., Fallas, M. A. y Vargas, J. (2010). Las competencias de educación del siglo XXI: un acercamiento a la realidad costarricense. Revista Electrónica Educere, 14(2), 187-205. DOI: https://doi.org/10.15359/ree.14-2.13

Gavidia, V. (Coord.) (2016). Los ocho ámbitos de la Educación para la Salud en la escuela. Valencia: Tirant Humanidades.

Giménez, L., Fernández, L., Mayoral, O. y Gavidia, V. (2016). Competencias en Promoción de la Salud. Análisis del currículo de la Educación Obligatoria. En V. Gavidia, Competencias en Salud (pp. 67-86). Valencia: Tirant Humanidades.

Herrera, A., et al. (2006). Proyecto de innovación en tutorías. Una experiencia desde la Facultad de Educación y Humanidades de Melilla en la Titulación de Maestro. En M. A. Gallardo et al. (Coords.), I Congreso Internacional de Psicopedagogía: Ámbitos de Intervención del Psicopedagogo (pp. 1-11). Granada: Universidad de Granada. 
López Ruiz, J. I. (2019). Crear unidades didácticas desde un enfoque de educación global. Un caso práctico. En J. M. Fernández Batanero y A. López Martínez (Coords.), Nuevos horizontes en educación. Innovaciones y experiencias (pp. 147-180). Barcelona: Octaedro.

Oliva, J. M. (2011). Dificultades para la implicación del profesorado de Educación Secundaria en la lectura, innovación e investigación en didáctica de las ciencias (I): el problema de la inmersión. Revista Eureka sobre enseñanza y divulgación de las ciencias, 8(1), 41-53. DOI: https://doi.org/10.25267/Rev_Eureka_ensen_divulg_cienc.2011.v8.i1.04

Olmedo, E. y Pegalajar, M. (2014). Teoría y principios psicopedagógicos que orientan la acción tutorial. En J. Expósito (Coord.), La acción tutorial en la educación actual. Madrid: Síntesis.

Pámies, M. y Galindo, C. (2015). Tutoría como herramienta académica del desarrollo de competencias transversales. Revista Iberoamericana de Educación, 68(1), 165-178. DOI: https://doi.org/10.35362/rie681179

Pérez de Eulate, L., Ramos, P., Liberal, S. y Latorre, M. (2005). educación nutricional: una encuesta sobre hábitos alimenticios en adolescentes vascos. Enseñanza de las Ciencias. Núm. extra. VII Congreso, 1-5.

Riquelme, M. (2006). Educación para la Salud escolar. Madrid: Exlibris.

Talavera, M. y Gavidia, V. (2007). Dificultades para el desarrollo de la Educación para la Salud en la escuela. Opiniones del profesorado. Didáctica de las Ciencias Experimentales y Sociales, (21), 119-128. DOI: https://doi.org/10.7203/dces.27.2569

Talavera, M. y Gavidia, V. (2013). Percepción de la Educación para la Salud en el personal docente y el sanitario. Didáctica de las Ciencias Experimentales y Sociales, (27), 115-129.

UNESCO (2016). Educación para la Ciudadanía Mundial. Preparar a los educandos para los retos del siglo XXI. París: UNESCO.

Valderrama, J. C., Gavidia, V., et al. (1997). Evolución de los hábitos de salud en la juventud de Valencia (1994-1996). Ayuntamiento Valencia. Programa Municipal de Drogodependencias. Plan Nacional de la Droga. 